\title{
Succinate Dehydrogenase-Deficient Renal Cell Carcinoma
}

National Cancer Institute

\section{Source}

National Cancer Institute. Succinate Dehydrogenase-Deficient Renal Cell Carcinoma. NCI

Thesaurus. Code C156464.

A very rare renal cell carcinoma that usually affects young adults. It is characterized by the presence of vacuolated, eosinophilic to clear neoplastic cells and loss of immunohistochemical expression of succinate dehydrogenase (SDH) B. In most cases germline mutations in an SDH gene (SDHA, SDHB, SDHC, or SDHD gene) are present. It has a relatively good prognosis. 